\title{
Psychosocial Factors Contributing to Suicidal Ideation in Hospitalized Schizophrenia Patients in Korea
}

\author{
Sung-Wan Kim ${ }^{1,2}$ \\ Su-Jung Kim ${ }^{3,4}$ \\ Ji-Woong Mun ${ }^{1,5}$ \\ Kyung-Yeol Bae ${ }^{1}$ \\ Jae-Min Kim ${ }^{1}$ \\ Seon-Young Kim ${ }^{1}$ \\ Su-Jin Yang ${ }^{1}$ \\ II-Seon Shin ${ }^{1}$ \\ Jin-Sang Yoon ${ }^{1}$ \\ ${ }^{1}$ Department of Psychiatry, \\ Chonnam National University \\ Medical School, Gwangju, \\ ${ }^{2}$ Gwangju Buk-gu \\ Mental Health Center, Gwangju, \\ ${ }^{3}$ Department of Psychiatry, \\ Naju National Hospital, Naju, \\ ${ }^{4}$ Rodem Clinic, Seoul, \\ ${ }^{5}$ Department of Psychiatry, \\ Yeong Gwang Shin-ha \\ Christian Hospital, \\ Yeonggwang, Korea
}

Objective This study aimed to comprehensively evaluate psychosocial risk factors associated with suicidality in patients with schizophrenia in Korea.

Methods The study sample consisted of 84 hospitalized patients with schizophrenia. Suicidal thoughts and a clear desire to be dead within 2 weeks were defined as a current suicidal ideation. Socio-demographic and clinical variables, including family history of completed suicides and psychiatric illnesses, were collected, and the Positive and Negative Syndrome Scale (PANSS), Calgary Depression Scale for Schizophrenia (CDSS), Beck Depression Inventory (BDI), Simpson-Angus Scale (SAS), Scale to assess Unawareness of Mental Disorder (SUMD), and Alcohol Use Disorders Identification Test (AUDIT) were administered to identify factors associated with a current suicidal ideation.

Results Forty-three subjects (51.2\%) reported clear suicidal ideation. Multivariate analysis revealed that later age of illness onset, previous suicide attempt, family history of completed suicide, depression, or substance abuse, fewer than one family visitation to the hospital per month, and score on the CDSS were independently related to current suicidal ideation in these subjects. Age, education level, and scores on the SUMD were not significantly associated with current suicidal ideation in the multivariate analysis, but were associated with suicidal ideation in a univariate analysis on the level of $\mathrm{p}<0.1$.

Conclusion The above clinical factors should be evaluated to predict and prevent suicidal risk in patients with schizophrenia. In particular, modifiable factors such as depression should be managed to reduce suicidality of hospitalized patients with schizophrenia.

Psychiatry Investig 2010;7:79-85

Key Words Schizophrenia, Suicidal ideation, Depression, Family, Korea.

Received: January 14, 2010 Revised: March 2, 2010 Accepted: April 14, 2010

Available online: May 4, 2010

\section{Introduction}

Suicide is the fourth leading cause of death in Korea. The Korean suicide rate is the highest of any member country of the Organisation for Economic Cooperation and Development (OECD). Therefore, suicide is regarded as one of the most serious public health and social issues in Korea. ${ }^{1}$ It is well known that more than $90 \%$ of all suicides are attributable to an emotional or psychiatric illness. ${ }^{2}$ Psychiatric diagnoses associated with completed suicide include major mood disorders, schizophrenia, and addiction disorders. ${ }^{3}$ Suicide is a major cause of death in patients with schizophrenia, and the risk of suicide among patients with schizophrenia is almost constantly 20 times higher than that seen in the general population. ${ }^{4} \mathrm{Up}$ to $50 \%$ of patients with schizophrenia are reported to attempt suicide at some time in their life. ${ }^{5}$ In particular, a large proportion of suicides among schizophrenia patients occurs during or shortly after hospitalization. ${ }^{6,7}$ It is, therefore, important to identify factors contributing to suicide risk for hospitalized patients with schizophrenia, in order to aid prevention.

Several studies have examined the association between suicide risk and clinical characteristics in patients with schizophrenia including male gender, age under 30 years, never-mar- 
ried marital status, depression, previous suicide attempts, history of substance abuse, repeated psychiatric hospitalizations, recent discharge from hospital, poor social support, and family history of suicide ${ }^{8-10}$ However, although trans-cultural and ethnic differences in suicide risk factors in patients with schizophrenia may exist, ${ }^{11,12}$ few attempts have been to identify cultural factors as determinants of suicidal behavior in this population. Furthermore, only a few studies have comprehensively evaluated the above variables in hospitalized patients with schizophrenia in Korea. In the present study, we examined the association between suicidality and the clinical and sociodemographic characteristics of schizophrenia patients hospitalized for chronic mental illness.

\section{Methods}

\section{Subjects}

All subjects were enrolled from the inpatient unit of a national mental hospital and met the DSM-IV ${ }^{13}$ criteria for schizophrenia. The subjects had been hospitalized for more than 1 month, and had received a stable dose of antipsychotic medication for a minimum of 2 weeks prior to the study for stabilization of symptoms. Subjects were excluded from this study if they had neurological disorders, serious medical illness, or mental retardation or were unable to comprehend and complete all questionnaires. All patients gave their written informed consent to participate and the protocols were approved by the institutional review board.

\section{Measures}

Each subject's suicidal ideations were evaluated. Suicidal thoughts and a clear intent to be dead within 2 weeks regardless of a concrete plan for suicide were defined as current suicidal ideation, while vague feelings of being tired of living were not defined as suicidal ideation. To ascertain factors associated with current suicidal ideation, various socio-demographic and clinical characteristics of subjects were evaluated as follows: age, gender, educational level, marital status, smoking, age at onset, duration of illness, type of antipsychotics administered (typical or atypical), chlorpromazine-equivalent dosage of current antipsychotics, ${ }^{14}$ history of previous suicide attempts, frequency of family visitations to the hospital during admission, and family (first and second degree) history of schizophrenia, depression, alcoholism, and completed suicide were recorded. Family history of completed suicide, depression, and alcoholism were combined as one variable as they had similar characteristics in terms of their relationship with suicide and as their relatively low prevalence would otherwise have resulted in a lack of statistical power. Personal history of attempted suicide and family history of completed suicide and psychiatric illness were obtained by interviewing a close family member as well as the subject.
Interrupted and aborted suicide attempts ${ }^{15}$ were included in the suicide attempt history.

Measures for psychopathology included the Positive and Negative Syndrome Scale (PANSS) ${ }^{16,17}$ to evaluate psychotic symptoms; the Calgary Depression Scale for Schizophrenia (CDSS), ${ }^{18,19}$ an objective measure with specificity for depressive symptoms in patients with schizophrenia; ${ }^{20}$ the Beck Depression Inventory (BDI), ${ }^{21,22}$ a subjective measure for depression; the Alcohol Use Disorders Identification Test (AUDIT), ${ }^{23,24}$ a self report test for screening of alcohol abuse; the SimpsonAngus Scale (SAS) ${ }^{25}$ for extrapyramidal symptoms; and the first three items of the Scale to assess Unawareness of Mental Disorder (SUMD) ${ }^{26}$ for general insight into their schizophrenia. Higher scores of the SUMD indicated poor insight. Alcohol abuse as a variable was defined by a score above 7 on the AUDIT. ${ }^{24}$

\section{Statistical analysis}

Subjects were divided into two groups according to current suicidal ideation. Socio-demographic and clinical characteristics were compared between the two groups using an independent t-test or chi-square test, as appropriate. To identify the interactions among variables that were related to suicidal ideation in the univariate analysis, Pearson correlation coefficients were calculated for continuous variables and independent $\mathrm{t}$-tests were conducted for categorical variables. Factors associated with current suicidal ideation in the univariate analyses $(\mathrm{p}<0.1)$ were entered simultaneously into multivariate models (logistic regression analyses) to assess the independent association between those factors and suicidality. An exploratory analysis was performed to investigate whether specific psychotic symptoms had any association with suicidal ideation; all PANSS items were compared according to the presence of suicidal ideation using an independent t-test. Data were analyzed using the SPSS program (version 13.0 for Windows). Statistical significance was defined as $p<0.05$ (two-tailed), while in the exploratory analysis it was defined as $\mathrm{p}<0.01$ (two-tailed) to avoid a Type I error resulting from multiple testing.

\section{Results}

A total of 84 subjects participated in the study. The sample included 45 men (53.6\%) and 39 women, with a mean age of $37.4 \pm 7.1$ (range 22-55) and a mean duration of illness of 12.9 \pm 6.1 . Of the participants, $43(51.2 \%)$ subjects had a current suicidal ideation. Comparisons of sociodemographic and clinical characteristics between the groups according to a current suicidal ideation are shown in Table 1.

\section{Univariate analysis}

Significant differences were found in the demographic and 
clinical characteristics of the two groups, specifically in their level of education, their age at the onset of schizophrenia, and their family history of completed suicide, depression, or alcoholism. Among the assessment scales, scores on the CDSS, the BDI, and general psychopathology of the PANSS were significantly higher in patients with suicidal ideation than in those without. Of the 30 items of the PANSS, only four items (G1 somatic concern, G2 anxiety, G3 guilt feelings, and G6 depression) showed significant positive associations with suicidal ideation (all p-value $<0.01$ ). Patients with suicidal ideation tended to be older, to have a history of previous suicide attempts, to receive fewer than one family visit to the hospital per month, and to have a higher total PANSS score and a lower SUMD score, although these factors did not reach statistical significance $(p<0.1)$. Gender, marital status, duration of illness, type and dose of administered antipsychotics, smoking, alcohol abuse, family history of schizophrenia, and score on the SAS were not associated with current suicidal ideation.

\section{Interactions between factors associated with suicidal ideation}

The correlation coefficients between variables that were associated with suicidal ideation in the univariate analysis are shown in Table 2. The SUMD score was negatively correlated with the CDSS and BDI scores. The scores on the BDI and general psychopathology of the PANSS were significantly correlated with the CDSS score.

Patients with fewer than one family visit per month were significantly older ( $39.5 \pm 6.2$ vs. $35.9 \pm 7.3 ; p=0.027)$ and had significantly higher BDI scores $(27.0 \pm 12.5$ vs. $19.4 \pm 11.4$; $\mathrm{p}=$ $0.006)$ than those with more than one family visit per month. Family history did not show any significant associations with other variables related to suicidal ideation.

\section{Multivariate analysis}

Odds ratios for associations with suicidal ideation in subjects were calculated by a logistic regression analysis adjusting for variables that were related to the suicidal ideation at a level of $\mathrm{p}<0.1$ in the univariate analysis (Table 3 ). Since the CDSS, BDI, and general psychopathology of the PANSS all represent depressive symptoms and they were significantly associated with each other, only the CDSS was entered into the model. The multivariate analysis revealed that later age of illness onset, previous suicide attempt, family history of

Table 1. Comparisons of sociodemographic and clinical characteristics between the groups according to a current suicidal ideation

\begin{tabular}{|c|c|c|c|}
\hline & $\begin{array}{l}\text { Suicide idea }(+) \\
\mathrm{N}=43(51.2 \%)\end{array}$ & $\begin{array}{l}\text { Suicide idea (-) } \\
\mathrm{N}=41(48.8 \%) \\
\end{array}$ & p-value \\
\hline Sex, male : female (\%) & $23: 20(51.3)$ & $22: 19(48.7)$ & 0.988 \\
\hline Age, mean (SD) years & $38.8(7.3)$ & $36.0(6.8)$ & 0.078 \\
\hline Educational level, mean (SD) years & $11.1(2.8)$ & $12.3(1.9)$ & 0.034 \\
\hline Divorced or bereaved, no: yes $(\%)$ & $28: 15(60.0)$ & $31: 10(40.0)$ & 0.293 \\
\hline Age at onset, mean (SD) years & $26.0(6.3)$ & $23.0(5.8)$ & 0.023 \\
\hline Disease duration, mean (SD) years & $12.7(5.4)$ & $13.0(6.9)$ & 0.843 \\
\hline Previous suicide attempts, no : yes (\%) & $22: 21(63.6)$ & $29: 12(36.4)$ & 0.066 \\
\hline Family history of schizophrenia, no : yes (\%) & $31: 12(50.0)$ & $29: 12(50.0)$ & 0.890 \\
\hline Family history of suicide, depression, or alcoholism, no : yes (\%) & $25: 18(69.2)$ & $32: 8(30.8)$ & 0.027 \\
\hline Fewer than one family visit/month, no : yes (\%) & $22: 20(64.5)$ & $29: 11(35.5)$ & 0.060 \\
\hline Alcohol abuse, no : yes $(\%)$ & $34: 9(56.3)$ & $34: 7(43.8)$ & 0.653 \\
\hline Smoking, no : yes $(\%)$ & $24: 19(57.6)$ & $27: 14(42.4)$ & 0.346 \\
\hline Antipsychotics, typical : atypical (\%) & $20: 23(51.1)$ & $19: 22(48.9)$ & 0.988 \\
\hline $\mathrm{CPZ}$ equivalent dose of antipsychotics, mean (SD) $\mathrm{mg}$ & $417.9(299.4)$ & $334.7(188.2)$ & 0.133 \\
\hline PANSS-positive, mean (SD) & $14.7(5.9)$ & $13.0(5.0)$ & 0.153 \\
\hline PANSS-negative, mean (SD) & $11.0(4.3)$ & $12.7(6.5)$ & 0.158 \\
\hline PANSS-general psychopathology, mean (SD) & $29.9(9.8)$ & $23.7(5.4)$ & 0.001 \\
\hline PANSS-total, mean (SD) & $55.6(17.2)$ & $49.2(13.6)$ & 0.063 \\
\hline BDI, mean (SD) & $28.3(10.8)$ & $15.3(10.3)$ & $<0.001$ \\
\hline CDSS, mean (SD) & $10.4(4.6)$ & $3.8(3.3)$ & $<0.001$ \\
\hline SAS, mean (SD) & $1.9(2.8)$ & $1.2(1.8)$ & 0.164 \\
\hline SUMD, mean (SD) & $4.6(2.0)$ & $5.5(2.3)$ & 0.065 \\
\hline
\end{tabular}

SD: standard deviation, CPZ: chlorpromazine, PANSS: Positive and Negative Syndrome Scale, BDI: Beck Depression Inventory, CDSS: Calgary Depression Scale for Schizophrenia, SAS: Simpson-Angus Scale, SUMD: Scale for Unawareness of Mental Disorder 
Table 2. Correlation coefficients among demographic and clinical characteristics associated with suicidal ideation in univarite analysis

\begin{tabular}{|c|c|c|c|c|c|c|c|}
\hline & Age & Age at onset & Education, year & SUMD & CDSS & BDI & PANSS, general \\
\hline Age at onset & $0.582^{* * *}$ & & & & & & \\
\hline Education, year & $-0.356^{* *}$ & $-0.356^{* *}$ & & & & & \\
\hline SUMD & -0.072 & -0.059 & 0.079 & & & & \\
\hline CDSS & 0.172 & 0.166 & -0.212 & $-0.365^{* *}$ & & & \\
\hline BDI & 0.279 & 0.210 & $-0.449^{* * *}$ & $-0.274^{* *}$ & $0.693^{* * *}$ & & \\
\hline PANSS, general & -0.027 & -0.074 & 0.140 & 0.039 & $0.435^{* * *}$ & 0.213 & \\
\hline PANSS, total & -0.058 & -0.135 & 0.157 & $0.231^{*}$ & 0.208 & 0.049 & $0.910^{* * *}$ \\
\hline
\end{tabular}

${ }^{*} \mathrm{p}<0.05,{ }^{* *} \mathrm{p}<0.01,{ }^{* * *} \mathrm{p}<0.001$. SUMD: Scale for Unawareness of Mental Disorder, CDSS: Calgary Depression Scale for Schizophrenia, BDI: Beck Depression Inventory, PANSS: Positive and Negative Syndrome Scale

Table 3. Predictors of suicidal ideation determined in the multivariate analysis

\begin{tabular}{lcc}
\hline Factors & OR* $(95 \%$ CI $)$ & p-value \\
\hline Age, year & $0.86(0.72,1.03)$ & 0.107 \\
Education, year & $0.94(0.63 .1 .40)$ & 0.756 \\
Age at onset, year & $1.38(1.08,1.75)$ & 0.009 \\
Previous suicide attempt, yes & $13.24(1.81,96.63)$ & 0.011 \\
Family history of suicide, depression, or alcoholism, yes & $10.91(1.58,75.48)$ & 0.015 \\
Family visitation during admission, $<1 /$ month & $11.62(1.27,105.94)$ & 0.030 \\
CDSS, score & $1.97(1.39,2.79)$ & $<0.001$ \\
SUMD, score & $0.87(0.52,1.46)$ & 0.872 \\
\hline
\end{tabular}

*adjusted for all other variables in the model. OR: odds ratio, CI: confidence interval, CDSS: Calgary Depression Scale for Schizophrenia, SUMD: Scale for Unawareness of Mental Disorder

suicide, depression, or alcoholism, fewer than one family visit per month, and score on the CDSS were independently associated with current suicidal ideation. Age, education lev$\mathrm{el}$, and scores on the SUMD were not significantly associated suicidal ideation in the multivariate analysis.

\section{Discussion}

In this study, we observed that suicidal ideation was common $(53.6 \%)$ in hospitalized patients with schizophrenia. Depression, previous suicide attempts, later age at onset of schizophrenia, family history of suicide, depression, or alcoholism, and fewer than one family visit per month were associated with the greater suicide risks in inpatients with schizophrenia.

Depression is an important risk factor for suicide in both the general population and in schizophrenia patients. ${ }^{27,28}$ In a review of 270 schizophrenic suicides, depressive symptoms were noted by their clinicians during the last period of contact before the suicide in approximately $60 \%$ of cases. ${ }^{29}$ Among the factors associated with suicidal ideation in this study, depression was the only modifiable factor in patients. In the thirty items of the PANSS, only four items, which were depression subfactors of the PANSS, ${ }^{30}$ were associated with suicidal ideation. Therefore, our study supports earlier reports suggesting the importance of effective management of depressive symptoms in schizophrenia to minimize suicide risks. ${ }^{31}$

The frequency of family visitations to the hospital during the admission period was used as a variable representing level of family support. The prevalence of suicidal ideation was significantly higher in patients with fewer than one family visit per month. Moreover, subjective depressive symptoms measured using the BDI were significantly more severe in these patients than in those with more than one family visit per month. These findings are also compatible with previous research, which has reported a correlation between poor social support and suicidality. ${ }^{32,33}$ A recent study on the suicidality of inmates in Korea also reported that the number of visitations by significant others was significantly related to suicide attempts. ${ }^{34}$ In Korean society, family is a more important source of environmental support than it is in Western society. Furthermore, the family plays a crucial role for schizophrenia patients because they tend to live with their family, according to Korean cultural tradition, rather than in residential facilities or individual homes, as in Western countries. ${ }^{35,36}$ The findings of this study support the view $^{31}$ that family support systems can be an important protective factor against suicidality in patients with schizophrenia.

Family history of psychiatric illness was also associated with increased suicidality of patients. In our subjects, high prevalence of schizophrenia in family members $(28.6 \%)$ was observed, confirming the hereditary factors in the pathogenesis 
of schizophrenia. However, suicidality did not differ according to family history of schizophrenia. Rather, a family history of suicide, depression, and alcoholism was significantly more frequent in patients with suicidal ideation than in those without. Earlier studies have reported that a family history of suicidal behavior is found significantly more often in schizophrenia patients who attempt or commit suicide than among patients who have never exhibited suicidal behavior. ${ }^{37-39}$ In a recent study on suicidality in those with first episode psychosis and in high-risk persons, psychiatric family history including affective disorder and substance abuse was associated with an enhanced risk of attempted suicide. ${ }^{40}$ Parents' substance abuse was also associated with adolescents' lifetime history of suicidal ideation. ${ }^{41}$ Both depression and substance abuse were related with low serotonergic activity and were some of the most important risk factors for suicide both in those with schizophrenia and in the general population. ${ }^{5,42,43}$ Therefore, genetic transmission of these psychiatric illnesses as a risk factor for suicide is one possible explanation for this finding. ${ }^{44}$ From a biological perspective, suicidality may be inherited independently from schizophrenia per se. However, environmental and nongenetic factors such as shared exposure to family stress and common lifestyles could contribute to the suicidal behavior of both patients and their family members. ${ }^{45}$

Previous attempted suicide in patients with schizophrenia is an important clinical indicator for both further attempts and completed suicide. ${ }^{31,46}$ In a previous study of Korean schizophrenia patients, a history of suicide attempts was predictive of current suicidality. ${ }^{47}$ In our previous study on a general population, patients who had previously attempted suicide were significantly more likely to have current suicidal ideation. ${ }^{48}$ The findings of this study are compatible with those of previous studies and suggest that patients with a history of suicide attempts should be managed with special attention aimed at preventing further suicidal behavior. In this study, later age at onset of schizophrenia was independently associated with current suicidal ideation. In previous studies, earlier age of illness onset was associated with suicidality in schizophrenia. ${ }^{49,50}$ Those studies suggested that an earlier age of illness onset was related with more severe psychopathology and a poorer prognosis, which might be associated with suicidality. However, recent studies carried out in Taiwan and Denmark identified a later age at onset of symptoms as one of the predictors of suicide risk in schizophrenia. ${ }^{51,52}$ Those studies suggested that developing a serious mental illness at a later age may make life more unbearable to the affected individual than is the case for those whose illness began earlier in life. Further studies are warranted to evaluate this relationship between suicidality and age of illness onset.

In previous studies, smoking and alcohol abuse were reported to be associated with suicidality in schizophrenia. ${ }^{31,53}$ However, results of the present study did not support those results.
The reason for the inconsistency may be that all subjects in this study were hospitalized and had limited access to cigarettes and alcohol.

Insight was a risk factor for current suicidality in the univariate analysis of the present study, but its significant association disappeared in a multivariate analysis. Previous studies have found that awareness of psychiatric condition was associated with increased risk of suicide, and this association was mediated by depression and hopelessness for the future. ${ }^{54-56}$ In our study, good awareness of the illness was associated with depression. Thus, clinicians should pay particular attention to feelings of demoralization when managing suicide risk in schizophrenic patients and try foster hopeful attitudes toward the future..$^{31,57}$

The strengths of our study are that comprehensive sociodemographic and clinical data including familial factors were obtained and well-validated assessment scales were used. However, this study has limitations that must be addressed when trying to generalize from the results. Recall bias might have influenced the information concerning family history. All subjects in the study were inpatients, and most of them were chronic patients. Nevertheless, our study confirmed that hospitalized chronic schizophrenia patients in Korea had similar risk factors for suicide as have been reported in previous studies. Moreover, we found out that familial factors, both social and biological, may contribute to suicidality in schizophrenia patients in Korea. In the future, a genetic study of schizophrenia patients and their family members should help to confirm biological risk factors for suicidality. Moreover, a longitudinal study to evaluate the effects of family intervention and the management of depression on reducing suicidality in schizophrenia patients is needed.

In conclusion, our study suggests that depression, previous suicide attempts, later age of illness onset, family history of suicide, depression, or alcoholism, and poor support system such as fewer than once family visitation per month may be related to suicidality in hospitalized schizophrenia patients. These factors should be evaluated to predict and prevent suicidal risk in patients with schizophrenia. Furthermore, modifiable factors such as depression and poor psychosocial support should be managed to reduce suicidality.

\section{- Acknowledgments}

This research was supported by a grant of the Korea Health 21 R\&D Project, Ministry of Health and Welfare, Republic of Korea (A050047).

\section{REFERENCES}

1. Hong JP, Bae MJ, Suh T. Epidemiology of suicide in Korea. Psychiatry Investig 2006;3:7-14.

2. Conwell Y, Duberstein PR, Cox C, Herrmann JH, Forbes NT, Caine ED. Relationships of age and axis I diagnoses in victims of completed suicide: a psychological autopsy study. Am J Psychiatry 1996;153: 1001-1008. 
3. Harris EC, Barraclough B. Suicide as an outcome for mental disorders. A meta-analysis. Br J Psychiatry 1997;170:205-228.

4. Nordentoft M, Laursen TM, Agerbo E, Qin P, Høyer EH, Mortensen PB. Change in suicide rates for patients with schizophrenia in Denmark, 1981-97: nested case-control study. BMJ 2004;329:261-264.

5. Perenyi A, Forlano R. Suicide in schizophrenia. Neuropsychopharmacol Hung 2005;7:107-117.

6. Hu WH, Sun CM, Lee CT, Peng SL, Lin SK, Shen WW. A clinical study of schizophrenic suicides. 42 cases in Taiwan. Schizophr Res 1991;5: 43-50.

7. Allebeck P, Wistedt B. Mortality in schizophrenia. A ten-year followup based on the Stockholm County Inpatient Register. Arch Gen Psychiatry 1986;43:650-653.

8. Breier A, Astrachan BM. Characterization of schizophrenic patients who commit suicide. Am J Psychiatry 1984;141:206-209.

9. Fenton WS. Depression, suicide, and suicide prevention in schizophrenia. Suicide Life Threat Behav 2000;30:34-49.

10. Hawton K, Sutton L, Haw C, Sinclair J, Deeks JJ. Schizophrenia and suicide: systematic review of risk factors. Br J Psychiatry 2005;187: 9-20.

11. Altamura AC, Mundo E, Bassetti R, Green A, Lindenmayer JP, Alphs $\mathrm{L}$, et al. Transcultural differences in suicide attempters: analysis on a high-risk population of patients with schizophrenia or schizoaffective disorder. Schizophr Res 2007;89:140-146.

12. Nam YY, Kim CH, Lee HS. Suicide and mental disorder: evidence of an increased risk of suicide in mental disorder and the risk factors associated with suicide. Psychiatry Investig 2006;3:36-50.

13. American Psychiatric Association. Diagnostic and Statistical Manual of Mental Disorders (4th ed). Washington, DC: American Psychiatric Press; 1994.

14. Woods SW. Chlorpromazine equivalent doses for the newer atypical antipsychotics. J Clin Psychiatry 2003;64:663-667.

15. Barber ME, Marzuk PM, Leon AC, Portera L. Aborted suicide attempts: a new classification of suicidal behavior. Am J Psychiatry 1998;155:385-389.

16. Kay SR, Fiszbein A, Opler LA. The positive and negative syndrome scale for schizophrenia. Schizophr Bull 1987;13:261-276.

17. Yi JS, Ahn YM, Shin HK, An SK, Joo YH, Kim SH, et al. Reliability and validity of the Korean version of the Positive and Negative Syndrome Scale. J Korean Neuropsychiatr Assoc 2001;40:1090-1105.

18. Addington D, Addington J, Schissel B. A depression rating scale for schizophrenics. Schizophr Res 1990;3:247-251.

19. Kim YK, Won SD, Lee KM, Choi HS, Jang HS, Lee BH, et al. A study on the reliability and validity of the Korean version of the Calgary Depression Scale for Schizophrenia (K-CDSS). J Korean Neuropsychiatr Assoc 2005;44:446-455.

20. Kim SW, Kim SJ, Yoon BH, Kim JM, Shin IS, Hwang MY, et al. Diagnostic validity of assessment scales for depression in patients with schizophrenia. Psychiatry Res 2006;144:57-63.

21. Beck AT, Ward CH, Mendelson M, Mock J, Erbaugh J. An inventory of measuring depression. Arch Gen Psychiatry 1961;4:561-571.

22. Hahn HM, Yum TH, Shin YW, Kim KH, Yoon DJ, Chung KJ. A standardization study of Beck Depression Inventory in Korea. J Korean Neuropsychiatr Assoc 1986;25:487-502.

23. Saunders JB, Aasland OG, Babor TF, de la Fuente JR, Grant M. Development of the Alcohol Use Disorders Identification Test (AUDIT): WHO Collaborative Project on Early Detection of Persons with Harmful Alcohol Consumption--II. Addiction 1993;88:791-804.

24. Lee BO, Lee CH, Lee PG, Choi MJ, Namkoong K. Development of Korean version of Alcohol Use Disorders Identification Test (AUDITK): its reliability and validity. J Korean Academy of Addiction Psychiatry 2000;4:83-92.

25. Simpson GM, Angus JWS. A rating scale for extrapyramidal side effect. Acta Psychiatr Scand 1970;212 Suppl:11-19.

26. Amador XF, Flaum M, Andreasen NC, Strauss DH, Yale SA, Clark
SC, et al. Awareness of illness in schizophrenia and schizoaffective and mood disorder. Arch Gen Psychiatry 1994;51:826-836.

27. Paykel ES. Depression: major problem for public health. Epidemiol Psichiatr Soc 2006;15:4-10.

28. Hawton K, Sutton L, Haw C, Sinclair J, Deeks JJ. Schizophrenia and suicide: systematic review of risk factors. Br J Psychiatry 2005;187:9-20.

29. Roy A. Relationship between depression and suicidal behavior in schizophrenia. In: De Lisi L, editor. Depression in schizophrenia. Washington, DC: American Psychiatric Press, 1990, p.39-58.

30. Kay SR, Sevy S. Pyramidal model of schizophrenia. Schizophr Bull 1990;16:537-545.

31. Roy A, Pompili M. Management of schizophrenia with suicide risk. Psychiatr Clin North Am 2009;32:863-883.

32. Westermeyer JF, Harrow M, Marengo JT. Risk for suicide in schizophrenia and other psychotic and nonpsychotic disorders. J Nerv Ment Dis 1991;179:259-266.

33. Bonner RL. Correctional suicide prevention in the year 2000 and beyond. Suicide Life Threat Behav 2000;30:370-376.

34. Kang BJ, Nam YY, Ko SM, Lee HS, Hong KE. The characteristics of suicide attempts and psychosocial risk factors in correctional institutions. J Korean Neuropsychiatr Assoc 2009;48:368-373.

35. Kim CK, Kim JW, Seo JM, Lee GZ, Kim GJ, Byun WT. Family burden of schizophrenics in the primary caregivers and siblings. J Korean Neuropsychiatr Assoc 2000;39:113-127.

36. Kim SW, Yoon JS, Choi SK. Survey of medication adherence in patients with schizophrenia--Korean ADHES data. Hum Psychopharmacol 2006;21:533-537.

37. Roy A. Family history of suicide. Arch Gen Psychiatry 1993;40:971974.

38. Wilkinson G, Bacon NA. A clinical and epidemiological survey of parasuicide and suicide in Edinburgh schizophrenics. Psychol Med 1984;14:899-912.

39. Qin P, Agerbo E, Mortensen PB. Suicide risk in relation to family history of completed suicide and psychiatric disorders: a nested casecontrol study based on longitudinal registers. Lancet 2002;360:11261130 .

40. Preti A, Meneghelli A, Pisano A, Cocchi A; Programma 2000 Team. Risk of suicide and suicidal ideation in psychosis: results from an Italian multi-modal pilot program on early intervention in psychosis. Schizophr Res 2009;113:145-150.

41. Pfeffer CR, Normandin L, Kakuma T. Suicidal children grow up: relations between family psychopathology and adolescents' lifetime suicidal behavior. J Nerv Ment Dis 1998;186:269-275.

42. Linnoila VM, Virkkunen M. Aggression, suicidality, and serotonin. J Clin Psychiatry 1992;53 Suppl:46-51.

43. Sher L. Alcoholism and suicidal behavior: a clinical overview. Acta Psychiatr Scand 2006;113:13-22.

44. Roy A. Family history of suicidal behavior and earlier onset of suicidal behavior. Psychiatry Res 2004;129:217-219.

45. Lee SI, Jung HY. Psychosocial risk factors for suicide. Psychiatry Investig 2006;3:15-22.

46. Pompili M, Lester D, Grispini A, Innamorati M, Calandro F, Iliceto P, et al. Completed suicide in schizophrenia: evidence from a case-control study. Psychiatry Res 2009;167:251-257.

47. Nam YY, Kim CH, Lee KH, Suh HS, Shin YJ, Sohn YH, et al. The effects of insight, depression, impulsivity, and past suicidal attempts on suicidality in schizophrenia. Schizophrenia Clinics 2003;6:158164.

48. Kim SW, Kim SY, Kim JM, Suh TW, Shin IS, Kim SJ, et al. A survey on attitude toward suicide and suicidal behavior in Korea. J Korean Soc Biol Ther Psychiatry 2008;14:43-48.

49. Gupta S, Black DW, Arndt S, Hubbard WC, Andreasen NC. Factors associated with suicide attempts among patients with schizophrenia. Psychiatr Serv 1998;49:1353-1355.

50. Müller DJ, Barkow K, Kovalenko S, Ohlraun S, Fangerau H, Kölsch 
$\mathrm{H}$, et al. Suicide attempts in schizophrenia and affective disorders with relation to some specific demographical and clinical characteristics. Eur Psychiatry 2005;20:65-69.

51. Kuo CJ, Tsai SY, Lo CH, Wang YP, Chen CC. Risk factors for completed suicide in schizophrenia. J Clin Psychiatry 2005;66:579-585.

52. Reutfors J, Brandt L, Jönsson EG, Ekbom A, Sparén P, Osby U. Risk factors for suicide in schizophrenia: findings from a Swedish population-based case-control study. Schizophr Res 2009;108:231-237.

53. Iancu I, Sapir AP, Shaked G, Poreh A, Dannon PN, Chelben J, et al. Increased suicidal risk among smoking schizophrenia patients. Clin Neuropharmacol 2006;29:230-237.

54. Bourgeois M, Swendsen J, Young F, Amador X, Pini S, Cassano GB, et al. Awareness of disorder and suicide risk in the treatment of schizo- phrenia: results of the international suicide prevention trial. Am J Psychiatry 2004;161:1494-1496.

55. Pompili M, Ruberto A, Kotzalidis GD, Girardi P, Tatarelli R. Suicide and awareness of illness in schizophrenia: an overview. Bull Menninger Clin 2004;68:297-318.

56. Crumlish N, Whitty P, Kamali M, Clarke M, Browne S, McTigue O, et al. Early insight predicts depression and attempted suicide after 4 years in first-episode schizophrenia and schizophreniform disorder. Acta Psychiatr Scand 2005;112:449-455.

57. Restifo K, Harkavy-Friedman JM, Shrout PE. Suicidal behavior in schizophrenia: a test of the demoralization hypothesis. J Nerv Ment Dis 2009;197:147-153. 